Gut, 1974, 15, 852-857

\title{
Oesophageal acid clearing: One factor in the production of reflux oesophagitis
}

\author{
C. STANCIU AND JOHN R. BENNETT \\ From the Gastrointestinal Unit, Hull Royal Infirmary, Kingston upon Hull
}

SUMMARY Oesophageal acid clearing has been measured by counting the number of swallows needed to raise the lower oesophageal pH from 1.5 to 5.0 after instilling $15 \mathrm{ml} 0.1 \mathrm{~N}$ hydrochloric acid. Normal subjects all had a result less than 12 , as did asymptomatic patients with hiatal hernias. Patients with symptomatic gastrooesophageal reflux tended to have abnormal acid clearance, as did those with oesophageal motility disorders. Acid clearance correlated well with the mean duration of spontaneous episodes of acid reflux during 15-hour continuous recordings of lower oesophageal pH.

Abnormal acid clearance was improved by raising the bedhead, by medical treatment for oesophagitis, and by metoclopramide.

We conclude that abnormal acid clearing may result from disturbed oesophageal motility and render patients with these disorders susceptible to reflux oesophagitis. However, it also seems that gastrooesophageal reflux may lead to impaired acid clearance, both by creating abnormal motility and by effects on the oesophageal wall, and thus lead to a 'vicious-spiral' oesophagitis. In treating gastrooesophageal reflux, attention must be paid to improving acid clearance.

In gastrooesophageal reflux, symptoms and the extent and severity of pathological change in the oesophageal mucosa may be partly determined by the duration of contact between gastric contents and the mucosa. This 'contact time' is related to the frequency and volume of reflux, but another factor, which has received little attention, is the speed with which the oesophagus returns errant gastric contents to the stomach.

Booth, Kemmerer, and Skinner (1968) described a test of oesophageal acid clearing, and we have used their technique to study this important oesophageal function further.

\section{Subjects and Methods}

We studied 89 subjects ( 48 male, 41 female) aged 17 to 71 years (mean 52.7 years). Diagnostically they fell into five groups: (1) 14 subjects without 'reflux' symptoms and with normal barium meals being investigated for functional bowel disorders; (2) four patients with hiatal hernias demonstrated by barium meal examination but no symptoms of gastrooesophageal reflux; (3) 11 patients with

Received for publication 7 August 1974. radiological signs of duodenal ulceration and symptoms of gastrooesophageal reflux also; (4) 55 patients with symptoms of gastrooesophageal reflux (substernal burning on lying or stooping, or on drinking hot fluids, associated with regurgitation of gastric contents)-12 had hiatal hernia and 18 gastrooesophageal reflux shown radiologically; (5) five patients with oesophageal motor abnormalities (two achalasia, three diffuse oseophageal spasm).

COMBINED MANOMETRIC AND PH RECORDING Three polyvinyl catheters were used, each lumen having an internal diameter of $1.2 \mathrm{~mm}$ and one lateral opening, $2.4 \mathrm{~mm}$ in length, near the sealed tip; the distance between the openings was $5 \mathrm{~cm}$. The recording tubes were connected to pressure transducers recording on a Devices M.19 multichannel direct-writing recorder. The catheters were constantly perfused with distilled water by means of a constant infusion syringe pump at a rate of 0.8 $\mathrm{ml} / \mathrm{min}$. Respiration was monitored by a belt pneumograph. Intraluminal $\mathrm{pH}$ was recorded by a Radiometer combined glass $\mathrm{pH}$ and reference electrode (GK 282C) with a Radiometer $\mathrm{pH}$ meter (type PHM 26), its output also being connected to the direct-writing recorder. 
After the assembly (catheters and $\mathrm{pH}$ electrode) had been introduced into the gastric fundus, with the patient supine, it was withdrawn in steps of $0.5 \mathrm{~cm}$. The resting pressure of the lower oesophageal sphincter was determined, as well as were resting pressure and swallowing waves in the distal oesophagus. Abnormal motor function of the body of the oesophagus was considered to be present when the oesophageal pressure rises in response to a swallow never exceeded $20 \mathrm{~cm}$ water and/or over $25 \%$ of pressure-wave responses to swallows were nonperistaltic.

\section{ACID CLEARING TEST}

Once the distal catheter opening and $\mathrm{pH}$ electrode were $5 \mathrm{~cm}$ above the lower oesophageal sphincter, perfusion of the catheters was stopped and $15 \mathrm{ml}$ of $0 \cdot 1 \mathrm{~N}$ hydrochloric acid was instilled through the proximal catheter, ie, $10 \mathrm{~cm}$ above the $\mathrm{pH}$ electrode; the $\mathrm{pH}$ of the distal oesophagus fell immediately to $1 \cdot 5$. The patients were told to swallow at 30 -second intervals and the total number of swallows necessary to raise the $\mathrm{pH}$ to 5.0 was counted. In 19 subjects (eight normal, 11 with gastrooesophageal reflux) the test was repeated with the bedhead raised $28 \mathrm{~cm}$. In 14 patients with gastrooesophageal reflux the test was repeated after a period of medical treatment (antacids two to three hourly, avoidance of stooping, bedhead elevation, and, where appropriate, weight reduction) varying from two weeks to one year. Finally, acid clearing was measured in 18 patients with gastrooesophageal reflux before and after a bolus intravenous injection of $10 \mathrm{mg}$ metoclopramide.

OVERNIGHT OESOPHAGEAL PH RECORDING Fifteen-hour recordings (6 pm to $9 \mathrm{am}$ ) of lower oesophageal pH were made, with the electrode $5 \mathrm{~cm}$ above the lower oesophageal sphincter. The exact level of the sphincter was known from the previous manometric studies, but in some patients the electrode position was also checked radiologically. The pH electrode was firmly anchored to the cheek with adhesive tape; continuous records were obtained. The pH electrode was tested in buffers of pH 4.0 and 6.5 before and after recording and no drift was found. Patients lay supine with one or two pillows, but with no restriction on movement during sleep. A 'reflux episode' was defined as any occasion on which oesophageal $\mathrm{pH}$ fell by at least $2 \mathrm{pH}$ units from the baseline $(6.0$ to 6.5$)$, and its duration as the time during which $\mathrm{pH}$ was below 5 . In each record the number of such episodes was counted and their mean duration determined. The proportions of total recording time during which the $\mathrm{pH}$ was less than 5 , 4 , and 3 were each calculated as percentages.

\section{ENDOSCOPY}

In 45 patients oesophagoscopy (using an Olympus EF or GIF-D2 endoscope) and mucosal biopsy were performed. Oesophagitis was classified as: $\mathbf{O}$ (normal endoscopic appearance, normal biopsy); A (mucosal reddening and/or hyperaemia endoscopically, and infiltration with neutrophils, plasma cells, and lymphocytes histologically); B (mucosa friable, bleeding, or ulcerated, and biopsy showing marked cellular infiltration of the lamina propria with ulceration).

\section{Results}

ACID CLEARING (FIG 1)

All normal subjects were able to raise the oesophageal $\mathrm{pH}$ to $5 \cdot 0$ or above with four to 12 swallows (mean 8.0 \pm SD 2.4). Usually the rise in pH commenced with the second swallow, but the $\mathrm{pH}$ change varied considerably from swallow to swallow (range 0 to $4.6 \mathrm{pH}$ units). There was no rise in $\mathrm{pH}$ between swallows. Figure 5 includes a tracing of normal acid clearing.

The patients with asymptomatic hiatal hernia had an acid clearing within the same range (mean $7.7 \pm$ SD $2 \cdot 5$ ) as the normal group.

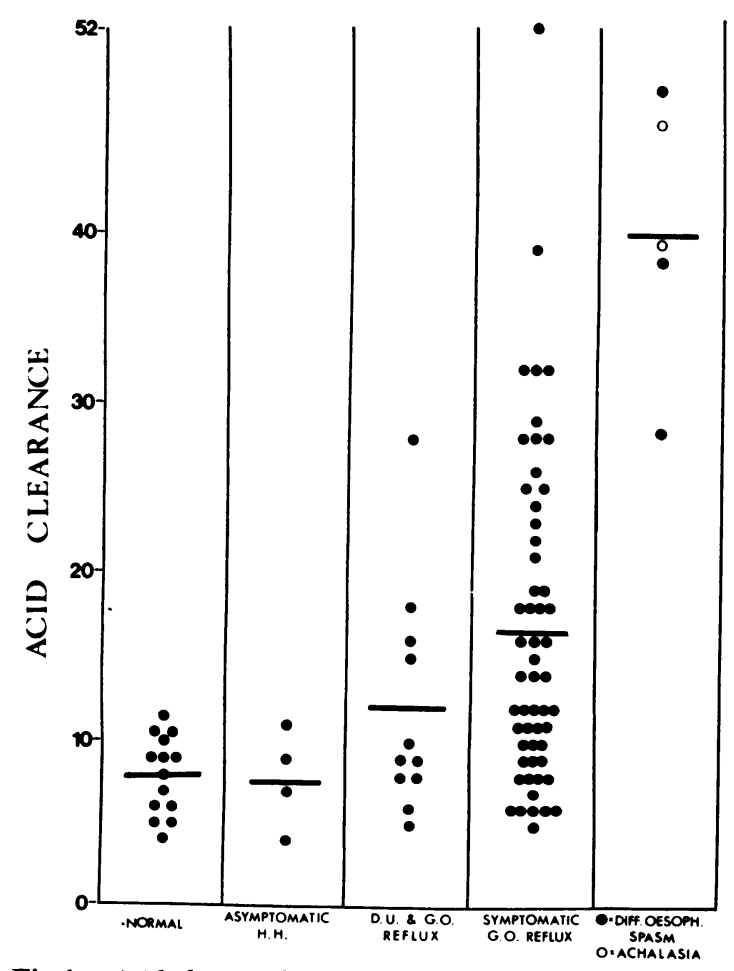

Fig 1 Acid clearing (number of swallows needed to raise lower oesophageal $\mathrm{pH}$ to 5.0 after acid load) in different groups of subjects. 
Four patients in group 3 (duodenal ulcer and gastrooesophageal reflux) had abnormal acid clearing (mean 12.0 \pm SD 6.4), but the difference between this group and normal subjects was not significant.

In patients with symptomatic gastrooesophageal reflux, the number of swallows necessary to raise the $\mathrm{pH}$ of the distal oesophagus to 5.0 varied widely: from 5 to 52 (mean 16.6 $\pm 9 \cdot 5$ ). In four patients oesophageal $\mathrm{pH}$ did not reach 5.0 after more than 30 swallows and the test was stopped because the patient could not tolerate it longer. Figure 6 includes a trace obtained from a patient with abnormal acid clearing. The difference between acid clearing in the normal group and patients with reflux was statistically significant $(P<0.001)$.

All patients in group 5 had abnormal acid clearing (mean 39.8 \pm SD 7.0), the difference from the normal group being statistically significant $(P<$ 0.001).

\section{ACID CLEARING AND OESOPHAGEAL} MOTILITY (FIG 2)

Of the 20 patients with abnormal oesophageal motility, all but two had prolonged acid clearing. However, many patients with delayed acid clearing had no abnormality of motility detectable by standard manometric studies, and two patients with manometrically abnormal motility cleared the acid load normally. The mean difference between those with normal and abnormal oesophageal motility, however, was significant $(P<0.001)$.

ACID CLEARING AND OESOPHAGITIS (FIG 3) As the degree of oesophagitis increased, oesophageal clearing deteriorated. Although the group with no

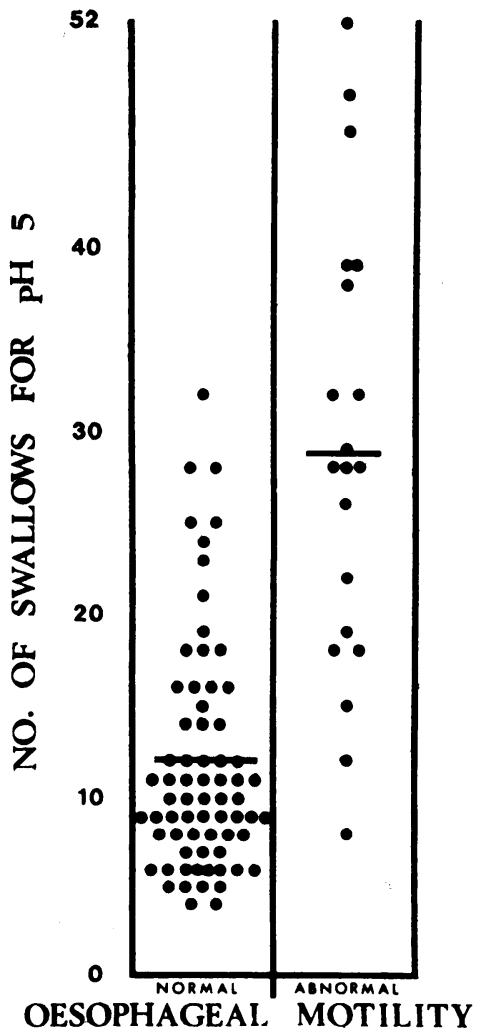

Fig 2

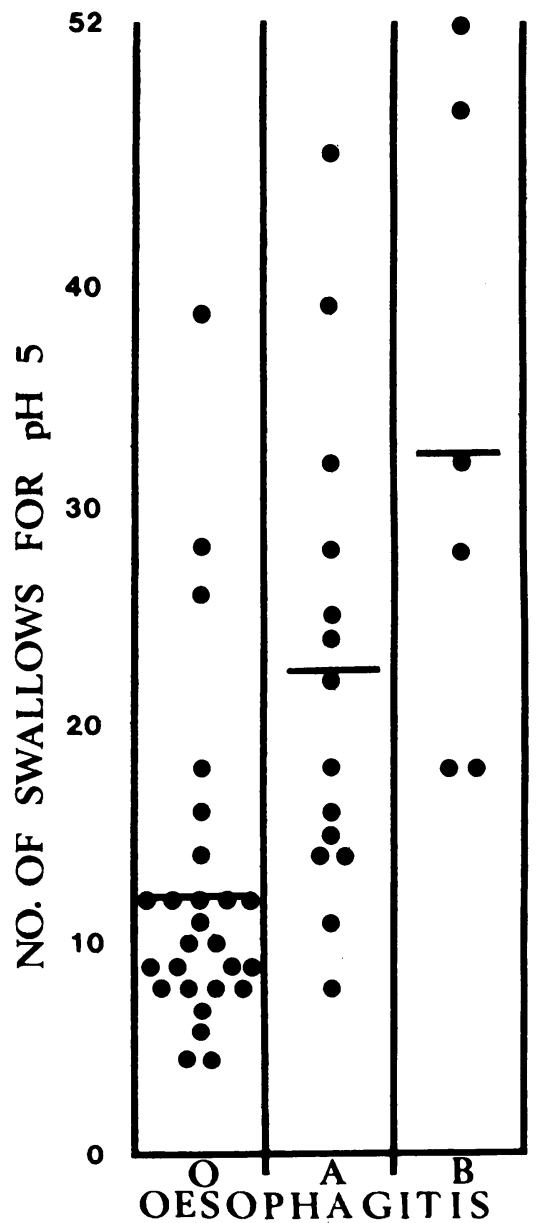

Fig 3
Fig 2 Acid clearance in subjects with manometrically normal and abnormal motility.

Fig 3 Acid clearance related to degree of oesophagitis. 


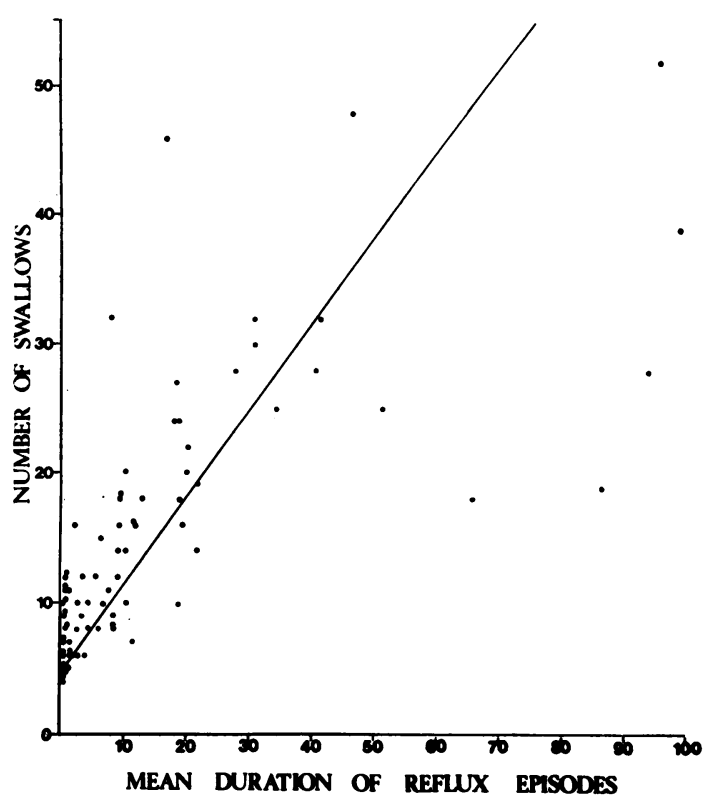

Fig 4 Correlation $(r=0 \cdot 71)$ between oesophageal acid clearing and mean duration of reflux episodes in 15-hour continuous lower oesophageal $\mathrm{pH}$ recordings $(n=79)$.

oesophagitis includes two patients with diffuse oesophageal spasm whose clearance was poor, there are significant differences between the mean value for those without oesophagitis and those with oesophagitis of grade A $(P<0.05)$ and grade B $(\mathrm{P}<0.01)$.
ACID CLEARING AND 15-HOUR RECORDING OF LOWER OESOPHAGEAL PH (FIG 4)

Acid clearing correlated with the mean duration of reflux episodes during 15 -hour oesophageal $\mathrm{pH}$ monitorings $(\mathrm{r}=0 \cdot 71)$. Figures 5 and 6 show comparative acid clearing tests and overnight $\mathrm{pH}$ recordings in two subjects.

EFFECTS OF THERAPY

Acid clearing increased significantly $(P<0.01)$ with the bedhead elevated (fig 7). Clearing improved in all but two patients after a course of medical treatment, and the mean result was significantly better $(\mathrm{P}<0.01)$ (fig 8). The two patients who showed no improvement had received only two weeks' treatment and we have not been able to test them subsequently.

Intravenous metoclopramide improved acid clearing, the mean number of swallows necessary to empty the oesophagus of the instilled acid being $16.3 \pm$ SD 5.6 before and $13.5 \pm 4.3$ after metoclopramide $(\mathrm{P}<0.01)$.

\section{Discussion}

It is known that gastrooesophageal reflux occurs at some time in most people (Spencer, 1969; Pattrick, 1970), though neither symptoms nor oesophagitis necessarily result. It is also recognized that the severity of reflux symptoms, the ease with which reflux occurs, and the degree of oesophagitis are poorly correlated (Tuttle, Bettarellu, and Grossman, 1960; Siegel and Hendrix, 1963; Ward, Wright, and Collis, 1970; Woodward, 1970), although the

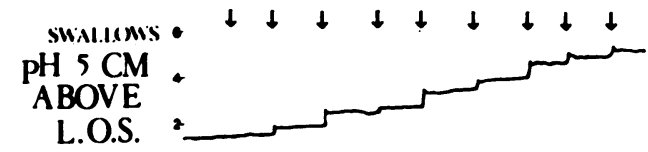

15 HOUR CONTINUOUS RECORDING OF LOWER OESOPHAGEAL $\mathrm{pH}$

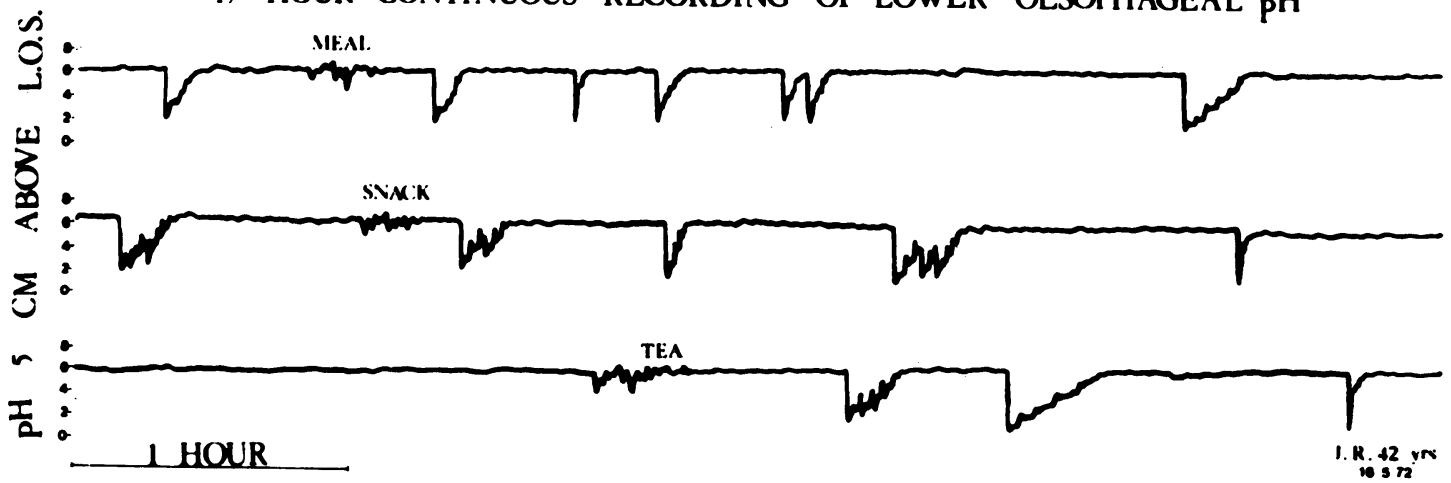

Fig 5 Recordings of acid clearing test (above) and 15-hour continuous lower oesophageal pH monitoring (below) in a normal subject. 


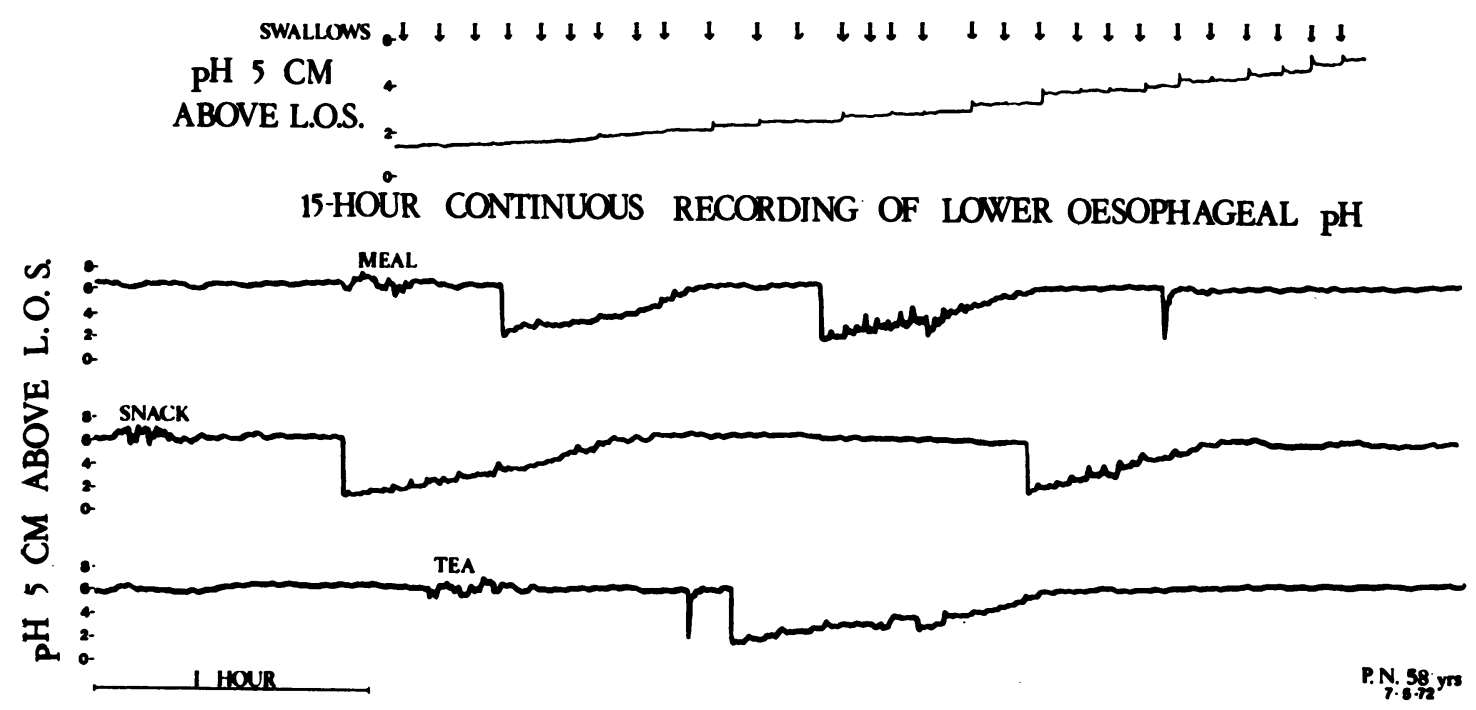

Fig 6 Recordings of acid clearing test (above) and 15-hour continuous lower oesophageal pH monitoring (below) in a patient with gastrooesophageal reflux.

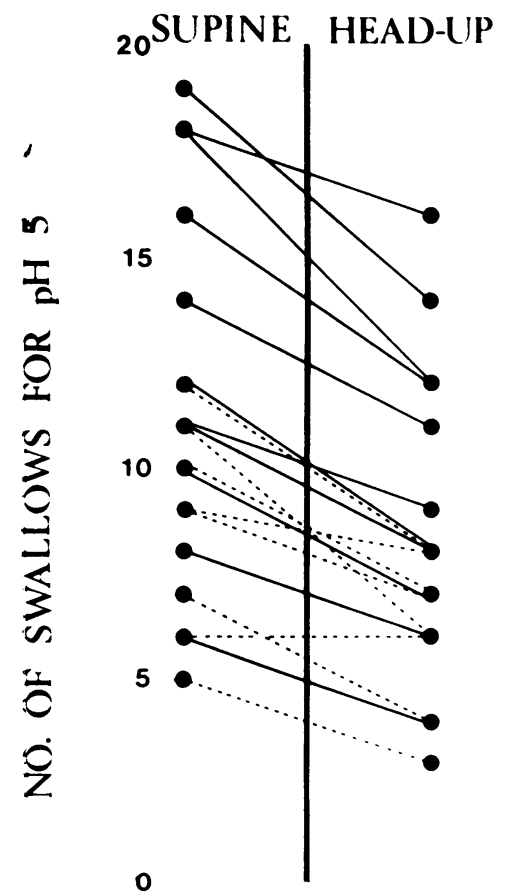

G.O. REFLUX NORMALS

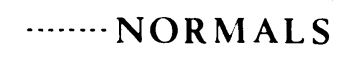

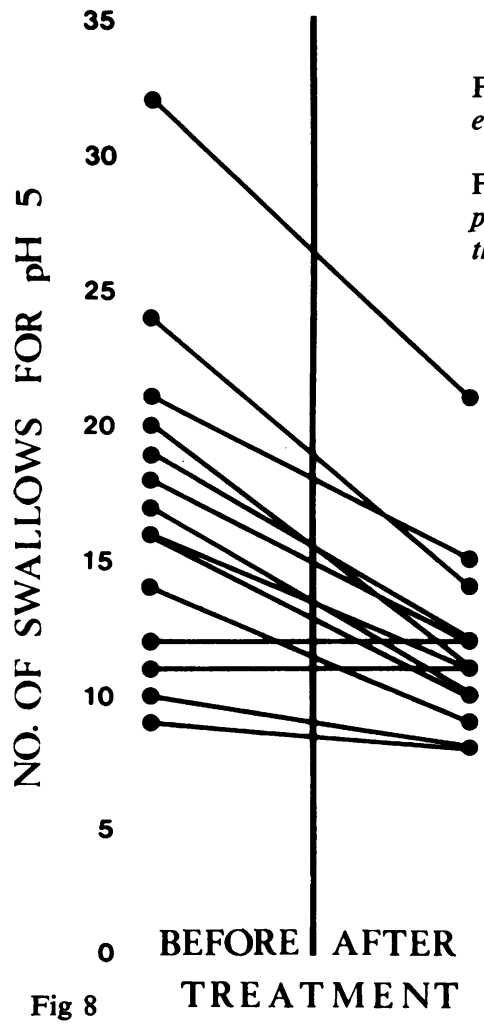

Fig 7 Change of acid clearing after elevation of bedhead by $28 \mathrm{~cm}$.

Fig 8 Change of acid clearing after period of medical therapy of not less than two weeks.

Fig 8 TREATMENT

Fig 7 
factors governing their relationships are not understood. Secondary peristalsis induced by a surge of refluxed gastric contents has been considered as a protective mechanism emptying the oesophageal lumen of irritant juices (Creamer, 1955; Nagler and Spiro, 1961).

It is conceivable that the efficiency with which the oesophagus empties refluxed material is one of the important factors in determining the results of gastrooesophageal reflux. It has been studied little, but the elegant device of assessing the disappearance of a standard acid bolus by $\mathbf{p H}$ recording, introduced by Booth et al (1968), affords a simple and reproducible way of so doing.

All our normal subjects 'cleared' an oesophageal load of $15 \mathrm{ml} 0 \cdot 1 \mathrm{~N}$ hydrochloric acid in 12 swallows or less, close to the maximum of 10 quoted by the originators of the test. Patients with asymptomatic hiatal hernias were within the same range, but patients with symptomatic gastrooesophageal reflux often showed delayed clearing.

That the performance of the oesophagus in this standardized test reflects its behaviour under normal physiological stress is demonstrated by the comparison of acid clearing with 15-hour lower oesophageal pH recordings. We believe that by monitoring the lower oesophageal $\mathrm{pH}$ over an extended period, without the artificial introduction of acid into the stomach, we can identify the spontaneous occurrence of gastrooesophageal reflux in an accurate and physiological way. It was interesting to find that the rate with which the oesophagus rid itself of an injected acid bolus correlated closely with the mean time taken for the lower oesophageal pH to return to normal after episodes of spontaneous acid reflux.

Efficiency of peristalsis might be expected to be a major factor in acid clearing, so it is not surprising that abnormal motility, detectable manometrically, was associated with delayed acid clearing. This probably accounts in part for the propensity to develop oesophagitis in systemic sclerosis and after treatment for achalasia by myotomy or forceful sphincter dilatation (Barlow, 1961; Ellis and Cole, 1965). However, delayed acid clearing was also found when manometry detected no abnormality of oesophageal peristalsis. This suggests that other factors, such as the physical state of the oesophageal mucosa, may influence propulsion of oesophageal contents, and possibly that there can be qualitative or quantitative motility changes too subtle to be detected by current manometric techniques.

Acid clearing was found to be poorer in patients with oesophagitis. Which is the primary abnormality? Does oesophagitis adversely affect the ability of the oesophagus to empty itself of acid within its lumen, or does the failure to clear refluxed acid lead to prolonged contact with the mucosa and consequent inflammation? Resolution of the question largely depends on the results of treatment. We found that acid clearance improved in $85 \%$ of patients after medical treatment, even though this only lasted two weeks in some cases. It therefore seems that reflux into a previously normal oesophagus may affect its physical properties, which will, in turn, diminish acid clearing, thus setting up a vicious spiral leading to established oesophagitis. In the treatment of gastrooesophageal reflux, therefore, attention must be paid not only to diminishing reflux but also to increasing oesophageal clearing. Our studies show that adequate elevation of the bedhead, antacid treatment, and the use of metoclopramide (Stanciu and Bennett, 1973) will all contribute to this aim.

This work was presented in part at the Odense Conference on the Function of the Esophagus, 1973.

Carol Stanciu was supported by a research grant from the Leeds Regional Hospital Board. The multichannel recorder was generously lent by Reckitt and Colman, Ltd. Invaluable technical help was given by Mrs Lynn Brocklesby, and secretarial assistance by Mrs M. Thomson. We are indebted to $\mathrm{Mr}$ W. Pridmore and Mr R. Hoare for statistical advice.

\section{References}

Barlow, D. (1961). Problems of achalasia. Brit. J. Surg., 48, 642-645. Booth, D. J., Kemmerer, W. T., and Skinner, D. B. (1968). Acid clearing from the distal esophagus. Arch. Surg., 96, 731-734.

Creamer, B. (1955). Oesophageal reflux. Lancet, 1, 279-281.

Ellis, F., and Cole, F. L. (1965). Reflux after cardiomyotomy. Gut, 6, 80-84.

Nagler, R., and Spiro, H. M. (1961). Heartburn in late pregnancy: manometric studies of esophageal motor function. J. clin. Invest., 40, 954-970.

Pattrick, F. G. (1970). Investigation of gastro-oesophageal reflux in various positions with a two-lumen pH electrode. Gut, 11, 659-667.

Siegel, C. I., and Hendrix, T. R. (1963). Esophageal motor abnormalities induced by acid perfusion in patients with heartburn. J. clin. Invest., 42, 686-695.

Spencer, J. (1969). Prolonged pH recording in the study of gastrooesophageal reflux. Brit. J. Surg., 56, 912-914.

Stanciu, C., and Bennett, J. R. (1973). Metoclopramide in gastrooesophageal reflux. Gut, 14, 275-279.

Tuttle, S. G., Bettarello, A., and Grossman, M. I. (1960). Esophageal acid perfusion test and a gastroesophageal reflux test in patients with esophagitis. Gastroenterology, 38, 861-872.

Ward, A. S., Wright, D. H., and Collis, J. L. (1970). The assessment of oesophagitis in hiatus hernia patients. Thorax, 25, 568-572.

Woodward, D. A. K. (1970). Response of the gullet to gastric reflux in patients with hiatus hernia and oesophagitis. Thorax, 25 , 459-464. 